\title{
Scanning Angle Raman Spectroscopy: A Nondestructive Method for Simultaneously Determining Mixed Polymer Fractional Composition and Film Thickness
}

Jonathan M. Bobbitt, Deyny Mendivelso-Perez, Emily A. Smith*

The Ames Laboratory, U.S. Department of Energy, and Department of Chemistry, Iowa State University, Ames, Iowa 50011, United States

Corresponding Author: *Email: esmith1@iastate.edu

\section{Keywords}

Vibrational Spectroscopy

Thin Polymer Films

Mixed Polymer Fractional Composition 
Abstract: A scanning angle (SA) Raman spectroscopy method was developed to simultaneously measure the chemical composition and thickness of waveguide mixed polymer films with varying fractional compositions. In order to test the method, six films of polystyrene-blockpoly(methyl methacrylate), some mixed with poly(methyl methacrylate) homopolymer (PS-bPMMA:PMMA), and two films of poly(2-vinylnapthalene)-block-poly(methyl methacrylate) (P2VN-b-PMMA) were prepared. The film thickness ranged from 495 to $971 \mathrm{~nm}$. The chemical composition and thickness of PS-b-PMMA:PMMA films was varied by the addition of the PMMA homopolymer and annealing the films in toluene. SA Raman peak amplitude ratios (1001 $\mathrm{cm}^{-1}$ for PS, $812 \mathrm{~cm}^{-1}$ for PMMA, and $1388 \mathrm{~cm}^{-1}$ for P2VN) were used to calculate the refractive index of the polymer film, an input parameter in calculations of the sum square electric field (SSEF). The film thickness was determined by SSEF models of the experimental Raman amplitudes versus the incident angle of light. The average film thickness determined by the developed SA Raman spectroscopy method was within 5\% of the value determined by optical profilometry. SA Raman spectroscopy will be useful for in situ label-free analyses of mixed polymer waveguide films.

\section{Introduction}

Analysis and characterization of polymer and mixed polymer films is important due to their increasing use in energy storage and capture devices, ${ }^{1-3}$ microelectronics, ${ }^{4-6}$ optics,,${ }^{7,8}$ and biomedical applications. ${ }^{9,10}$ The chemical composition and thickness of the films are important to device performance, and for accurately modeling and optimizing new devices before fabrication. Ellipsometry is a common noninvasive optical-based technique that measures the thickness of polymer films, but the technique does not provide chemical content information. ${ }^{11,12}$ Other 
methods for measuring polymer film thicknesses are profilometry and atomic force microscopy (AFM) ${ }^{13}$ Profilometry and AFM require a probe to be in contact with the sample surface and for the film to be scratched in order to measure the film thickness, respectively.

Scanning electron microscopy (SEM) and transmission electron microscopy (TEM) are capable of producing high spatial resolution images of polymer and mixed polymer films, and with the use of heavy metal staining different chemical components can be distinguished. ${ }^{14-17}$ SEM provides information on the film surface, but does not provide information from the bulk of the film. TEM provides higher resolution images compared to SEM, but requires more rigorous sample preparation. TEM is used to study the internal structure of a sample by showing regions of high or low electron density due to the number of electrons transmitted through the sample. Secondary ion mass spectrometry can also provide information on the internal structure and thickness of the film when operated in a depth profiling mode. ${ }^{18,19}$ All of the above characterization techniques provide high resolution information; however, the techniques are sample destructive and are not well suited for in situ measurements.

Vibrational spectroscopy is nondestructive and provides information on chemical structure. Attenuated total reflection infrared spectroscopy is capable of measuring polymer film thicknesses with a spatial resolution of $10 \mu \mathrm{m}$ and providing chemical content information; however, the evanescent wave penetration depth varies across the spectrum complicating the data analysis. ${ }^{20-23}$ Raman spectroscopy is an inelastic optical scattering effect that relies on the polarizability of a molecule. Raman spectroscopy using conventional illumination geometries (e.g., epi-illumination) cannot simultaneously measure the fractional composition and film thickness, and is not well suited for films with thicknesses in the hundreds of nanometer regime. Scanning angle (SA) Raman spectroscopy is a nondestructive technique that has been used to 
measure thickness and buried interfaces of thin polymer films. ${ }^{24-26}$ It can be used for polymer films of tens of nanometers to a few microns in thickness. A SA Raman dataset consist of Raman amplitudes as a function of Raman shift and incident angle for a sample optically interfaced to a prism as shown in Figure 1.

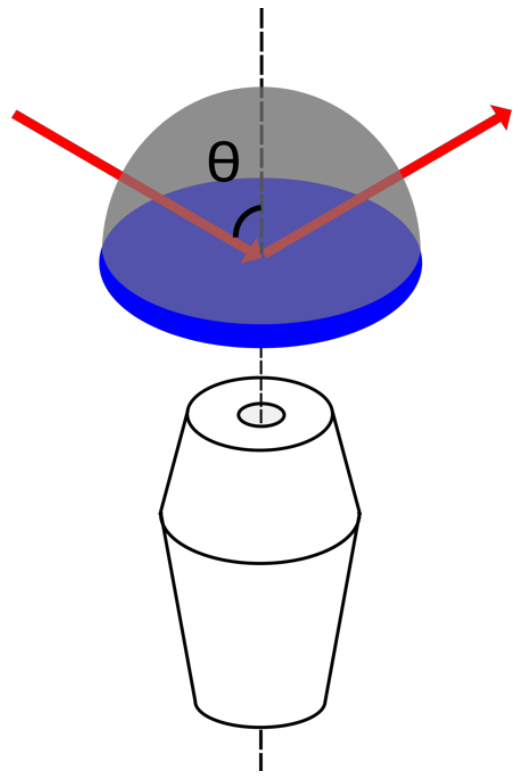

Figure 1: Schematic of the sample setup for SA Raman spectroscopy where the light (red arrow) is coupled through a prism (gray hemisphere) to generate the Raman signal as the incident angle of light is scanned. This signal is collected by a $10 \times$ microscope objective from below the sample. The blue layer represents the polymer film sample.

A dielectric material (e.g., polymer film) will act as a waveguide when the film thickness is greater than $\frac{\lambda}{2 \eta}$, where $\lambda$ is the wavelength of light and $\eta$ is the refractive index for the dielectric. Light coupled into a waveguide via a prism will be confined to propagate within the film and will exhibit constructive or destructive interference at various incident angles, which are recorded in the Raman spectra collected at varying incident angles. Plots of the Raman peak amplitude versus incident angle show the waveguide mode(s). The waveguide mode location and 
full width at half maximum values (fwhm) can be modeled with sum square electric field (SSEF) calculations when the refractive index or film thickness is known.

Outlined herein is a nondestructive method using SA Raman spectroscopy combined with SSEF calculations to extract the fractional composition and thickness of mixed polymer waveguide films. This work extends previous studies of homopolymer films reported by Meyer et al. that used SA Raman spectroscopy to measure film thickness when the index of refraction was known. ${ }^{24}$ Herein, the method was demonstrated using thin polystyrene-block-poly(methyl methacrylate) (PS-b-PMMA) films, PS-b-PMMA with added PMMA homopolymer (PS-bPMMA:PMMA) mixed films, and poly(2-vinylnapthalene)-block-poly(methyl methacrylate) (P2VN-b-PMMA) films. PS-b-PMMA and P2VN-b-PMMA are block copolymers that consist of two or more chemically dissimilar homopolymer (blocks) that are covalently attached. Block copolymers have been previously implemented in nanolithography to create masks for a variety of applications ranging from circuit and nanowire array fabrication to homogenous nanocluster arrays for surface-enhanced Raman scattering substrates. ${ }^{27-31}$ In the present study, these block copolymers are used to make mixed polymer samples with an unknown refractive index and thickness. The demand for nondestructive techniques that measure thin film thickness is continually increasing, and SA Raman spectroscopy is capable of providing this information plus chemical content information on complex polymer mixtures. The method has the capacity to provide real-time (dynamic) measurements during film preparation and post-processing conditions.

\section{Materials and Methods}


2.1 Sample Preparation. A $120 \mathrm{mg} / \mathrm{mL}$ stock solution of PS-b-PMMA (21500-b-7900 $\mathrm{g} / \mathrm{mol}$ with a polydispersity index (PDI) of 1.10, Polymer Source, Montréal, Canada) prepared in toluene (Fisher Scientific, Waltham, MA) and a stock solution of $120 \mathrm{mg} / \mathrm{mL}$ PMMA (120,000 $\mathrm{g} / \mathrm{mol}$ with a PDI of 2.0, Sigma Aldrich, St. Louis, MO) in toluene were prepared. The stock solutions were used to prepare 1:2, 1:2.4, 1:2.6, 1:3, and 1:4 (v/v) PS-b-PMMA:PMMA solutions. A $120 \mathrm{mg} / \mathrm{mL}$ stock solution of P2VN-b-PMMA in toluene (53000-b-140000 g/mol with a PDI of 1.20, Polymer Source, Montréal, Canada) was used to prepare 133 and $97 \mathrm{mg} / \mathrm{mL}$ solutions. To determine the refractive index of P2VN a $120 \mathrm{mg} / \mathrm{mL}$ solution of P2VN (PDI of 2.9, Polymer Source, Montréal, Canada) was prepared in toluene. PS-b-PMMA, PS-bPMMA:PMMA, P2VN-b-PMMA, and P2VN films were prepared by spin coating $200 \mu \mathrm{L}$ of solution onto $25 \mathrm{~mm}^{2}$ glass cover slips (Corning Inc., Corning, NY) at $3000 \mathrm{rpm}$ for 60 seconds using a KW-4A spin coater (Chemat Technology, Northridge, CA). The polymer film was placed in a $180 \mathrm{~cm}^{3}$ glass vessel with $500 \mu \mathrm{L}$ of acetone added as an annealing solvent, and kept at room temperature $\left(20^{\circ} \mathrm{C}\right)$. After annealing for 60 minutes, the films were removed from the annealing vessel to let the solvent vapors fully evaporate from the film. The film thicknesses were measured after completing the SA Raman and fluorescence measurements with a NewView 7100 optical profilometer (Zygo, Middlefield, CT). A sharp needle was used to make three scratches in the center of each film in order to determine the average thickness.

The underlying substrate (i.e., glass or sapphire) affects the properties of the PS-rich and PMMA-rich areas (data not shown). All polymer films were prepared on glass slides to enable characterizing them with several analysis methods, and the wedge transfer method was used to transfer the films to $25.4 \mathrm{~mm}$ diameter sapphire disks (Meller Optics, Providence, RI) for SA Raman measurements. ${ }^{32}$ Sapphire prisms and substrates were used for the SA Raman 
measurements to reduce background in the spectra. ${ }^{33}$ Figure S1 shows fluorescence images of a 1:2 PS-b-PMMA:PMMA film prepared on a glass substrate and after transferring it to a sapphire substrate. The images show no discernible changes in the polymer film after the transfer.

2.2 Raman Measurements. The SA Raman spectra were collected on a home-built instrument, previously described by Mckee et al., ${ }^{33}$ where a S-polarized 785-nm laser (Toptica Photonics, Victor, NY) was directed onto a sapphire prism using a galvanometer mirror on a translational stage. The instrument has an angle range of 25.00 to $75.00^{\circ}$ with an angular resolution of $0.05^{\circ}$. A $10 \times$ objective with a 0.22 numerical aperture (Nikon, Melville, NY) was used to collect the Raman signal and direct it to the side port of the optical microscope (Nikon, Melville, NY), where the light was focused onto a f/1.8i Holospec imaging spectrometer (Kaiser Optical Systems, Ann Arbor, MI). The spectra were collected with a Pixis 400BR charged coupled device $(\mathrm{CCD})$ with $1300 \times 400$ pixels (Princeton Instruments, Trenton, NJ) for 60 seconds. Two replicate measurements were collected at each angle from $50.0^{\circ}$ to $60.0^{\circ}$ using a $0.5^{\circ}$ step size, except around the waveguide maximum where a step size of $0.2^{\circ}$ was used.

Epi-illumination Raman spectra were collected using an XploRA Plus confocal Raman microscope (Horiba Scientific, Edison, NJ) and a 785-nm excitation source. The relative Raman cross-section ratios between PS/PMMA and P2VN/PMMA were determined by melting solid PS, PMMA, and P2VN onto glass substrates. The film thickness was $1.8 \pm 0.3,2.7 \pm 0.3$, and 2.5 $\pm 0.2 \mathrm{~mm}$ for PS, PMMA, and P2VN, respectively. These thicknesses were sufficient to acquire Raman amplitudes that were independent of the optical focus. Epi-illumination spectra were collected for 5 seconds from 3 different spots on the film. The relative Raman cross-section ratio $\left(\sigma_{R}\right)$ was calculated using equation 1 , where $I$ represents the indicated peak amplitude.

$$
\sigma_{R}=\frac{I_{P S, 1001 \mathrm{~cm}^{-1} \text { or } P 2 V N, 1388 \mathrm{~cm}^{-1}}}{I_{P M M A, 812 \mathrm{~cm}^{-1}}}=4.1(P S \text { to PMMA) and } 7.0(P 2 \mathrm{VN} \text { to PMMA) }
$$


The relative Raman cross-section ratio was used in the calculation of the Raman amplitude ratio (r) from the SA Raman data according to equations 2 and 3.

$$
\begin{aligned}
& r_{P S}=\frac{I_{P S, 1001 \mathrm{~cm}^{-1} \text { or } P 2 V N, 1388 \mathrm{~cm}^{-1}}}{I_{P S, 1001 \mathrm{~cm}^{-1} \text { or } P 2 V N, 1388 \mathrm{~cm}^{-1}}+\left(I_{P M M A, 812 \mathrm{~cm}^{-1}} \times \sigma_{R}\right)} \\
& r_{P M M A}=\frac{I_{P M M A, 812 \mathrm{~cm}^{-1}} \times \sigma_{R}}{I_{P S, 1001 \mathrm{~cm}^{-1} \text { or } P 2 V N, 1388 \mathrm{~cm}^{-1}}+\left(I_{P M M A, 812 \mathrm{~cm}^{-1}} \times \sigma_{R}\right)}
\end{aligned}
$$

The index of refraction of P2VN at $785 \mathrm{~nm}$ was not found in the literature. The index of refraction of $\mathrm{P} 2 \mathrm{VN}$ homopolymer was measured as reported in the supplemental information (Figure S2).

All data were processed using Igor Pro 6.37 scientific analysis and graphing software. The spectra were fit to a Gaussian function with a linear baseline using the batch-fit function in order to extract the peak amplitudes. Matlab 2015b was used to plot the SA Raman spectra as a function of their incident angle.

2.3 Sum Square Electric Field Calculations. Finite-difference time-domain simulations were used to calculate the SSEF (EM Explorer, San Francisco, CA) within the PS-b-PMMA, PSb-PMMA:PMMA, and P2VN-b-PMMA films. The inputs needed for the calculations were the refractive index and the thickness for the sapphire prism and polymer film. The refractive index at 785-nm for PS (1.579), PMMA (1.485), P2VN (1.620), and sapphire (1.753) using S-polarized light were found in the literature. ${ }^{34-36}$ An angle range of $50.00^{\circ}$ to $60.00^{\circ}$ was used for the calculations with a resolution of $0.05^{\circ}$ to ensure enough data points were calculated around the waveguide maximum. The Yee cell size was set to $5 \mathrm{~nm}$ to provide a compromise between highest accuracy and shortest computational time.

2.4 Fluorescence Microscopy Measurements. A $20 \mathrm{nM}$ solution of Rhodamine 6G was prepared in methanol, and $20 \mu \mathrm{L}$ was drop cast onto the PS-b-PMMA and PS-b-PMMA:PMMA 
films after the SA Raman measurements were complete. The films were dried for 10 minutes before images were acquired to allow the methanol to evaporate. Fluorescence images were collected at room temperature using an inverted microscope (Nikon Eclipse, TE2000U, Melville, NY, USA) equipped with a 60× air objective (Nikon Plan Fluor, 0.95 numerical aperture) and an excitation (Chroma HQ500/40, $500 \pm 40 \mathrm{~nm}$ ) and emission (Chroma HQ620/60m, $620 \pm 60 \mathrm{~nm}$ ) filter set for Rhodamine 6G. A mercury lamp was used as an illumination source and the images were captured using a PhotonMAX 512 EMCCD camera (Princeton Instruments, Trenton, NJ, USA) with a 300 millisecond acquisition time.

\section{Results and Discussion}

\subsection{Preparation and Preliminary Characterization of the Polymer Samples used to}

Demonstrate the SA Raman Method. Polymer waveguide films are made from block coplymer or block copolymer mixed with homopolymer. The amount of PMMA homopolymer added to the PS-b-PMMA:PMMA solution is varied from 1:2 to 1:4 to produce films with varying polymer fractional composition and thickness. Fluorescence and epi-Raman imaging are used to independently verify the fractional composition of the films. There is no observable phase segregated domains in the fluorescence image of a PS-b-PMMA film (figure 2A) without added homopolymer, likely due to the domains being smaller than the diffraction limit of light. However, the addition of PMMA homopolymer increases the size of the phase segregated domains, and they are observable by fluorescence microscopy (Figure 2B-F).

The chemical identities of the phases measured by fluorescence are determined using epiillumination Raman imaging (Figure S3). The high fluorescence intensity regions contain 
primarily PMMA with $8.6 \pm 0.2 \%$ PS. The low fluorescence intensity regions contain primarily PS with $32.9 \pm 0.3 \%$ of PMMA.

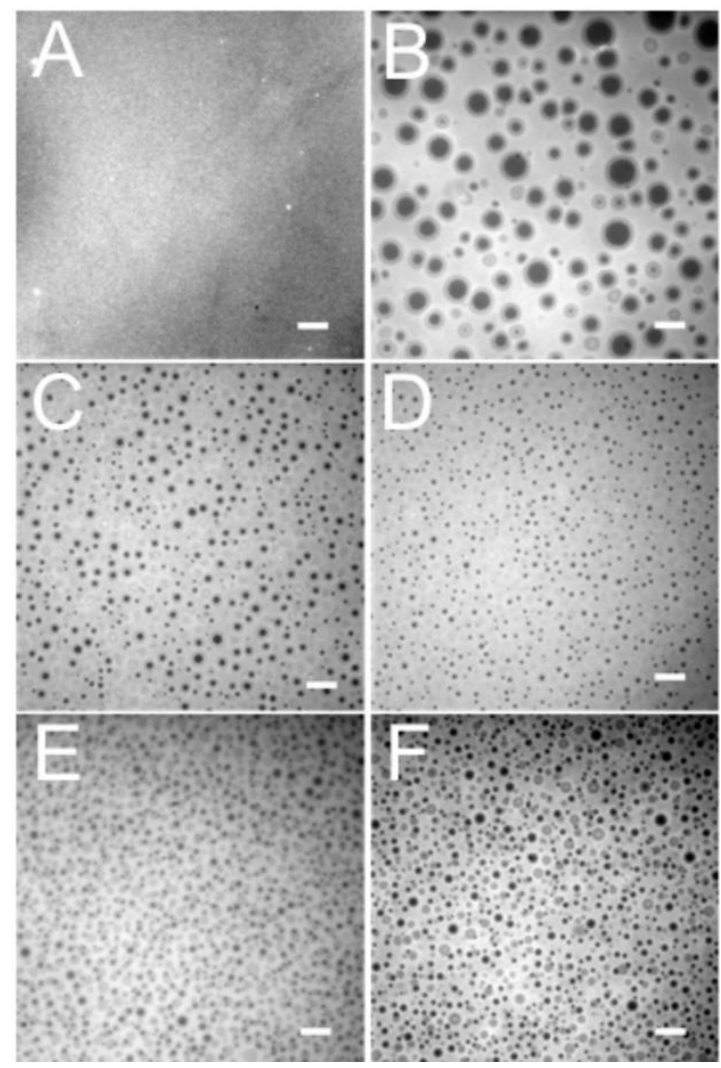

Figure 2: Fluorescence images collected with a $60 \times$ objective with the low fluorescence regions corresponding to PS-rich areas and the high fluorescence regions corresponding to PMMA-rich areas. (A) shows the PS-b-PMMA film. (B) shows the 1:2 PS-b-PMMA:PMMA film with a fluorescence area ratio of $0.27 \pm 0.02$. (C) shows the 1:2.4 PS-b-PMMA:PMMA film with a fluorescence area ratio of $0.21 \pm 0.01$. (D) shows the 1:2.6 PS-b-PMMA:PMMA film with a fluorescence area ratio of $0.181 \pm 0.002$. (E) shows the 1:3 PS-b-PMMA:PMMA film with a fluorescence area ratio of $0.180 \pm 0.007$. (F) shows the 1:4 PS-b-PMMA:PMMA film with a fluorescence area ratio of $0.170 \pm 0.007$. The scale bar is $9 \mu \mathrm{m}$ for all images. 


\subsection{Development of a SA Raman Method for Determining Fractional Composition}

and Film Thickness. A $0.12 \mathrm{~mm}^{2}$ area is illuminated in the SA Raman measurements. This is an area larger than the full images shown in Figure 2, so multiple PS-rich domains and a large PMMA-rich area are measured. The SA Raman data for the PS-b-PMMA film and five PS-bPMMA:PMMA films with varying amounts of added homopolymer are plotted in the left column of Figure 3 as their Raman shift versus the incident angle with the color scale representing the Raman amplitudes. All of the polymer films are plotted on the same color scale ranging from 500 to 8000 counts. 

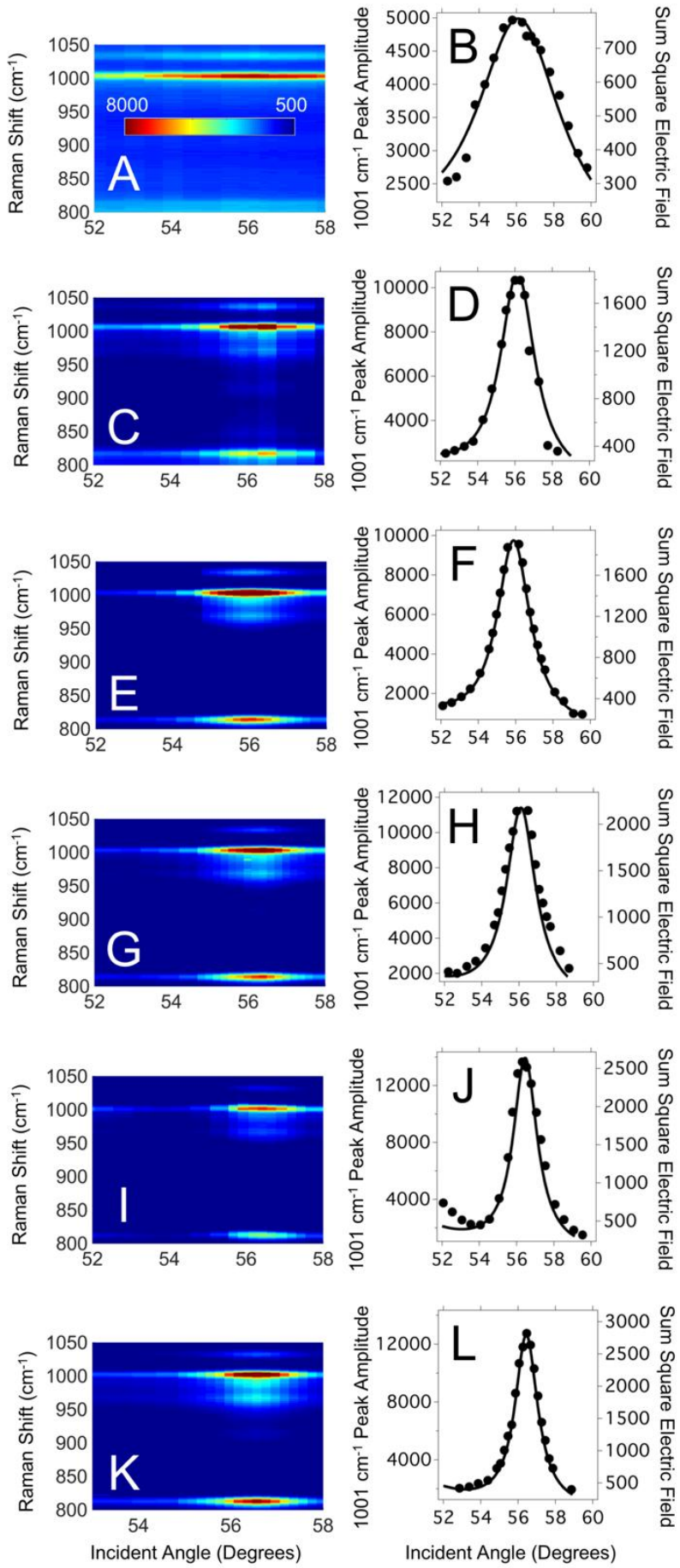

Figure 3: (A, C, E, G, I, and K) SA Raman spectra of PS-b-PMMA and PS-b-PMMA:PMMA films plotted on the same color amplitude scale shown in (A). (B, D, F, H, J, and L) show the $1001 \mathrm{~cm}^{-1}$ PS peak amplitude versus incident angle spectra and the solid black line is the SSEF 
fit for each sample. (A and B) PS-b-PMMA, (C and D) 1:2 PS-b-PMMA:PMMA, (E and F) 1:2.4 PS-b-PMMA:PMMA, (G and H) 1:2.6 PS-b-PMMA:PMMA, (I and J) 1:3 PS-bPMMA:PMMA, and (K and L) 1:4 PS-b-PMMA:PMMA films.

Figure 3A shows the SA Raman spectra at all collected incident angles for the PS-bPMMA film without added homopolymer. The peaks assigned to PS $\left(1001 \mathrm{~cm}^{-1}\right)$ and PMMA $\left(812 \mathrm{~cm}^{-1}\right)$ show a broad waveguide mode from approximately 52.3 to 59.8 degrees. The PMMA peak at $812 \mathrm{~cm}^{-1}$ has a significantly lower amplitude compared to the PS peak at $1001 \mathrm{~cm}^{-1}$. This is because there is $2.7 \times$ less PMMA in the PS-b-PMMA film, and also due to PMMA's smaller Raman cross-section compared to PS. The Raman amplitude ratio ( $\left.\mathrm{r}_{\mathrm{PS}}\right)$, as defined by equation 2 , for the PS-b-PMMA film is $0.78 \pm 0.01$. This is consistent with the manufacturer's reported 0.73 monomer fraction for this block copolymer film.

Figure 3B shows the cross section of the $1001 \mathrm{~cm}^{-1}$ PS peak observed in Figure 3A, where the amplitude (black circles) is plotted versus the incident angle for the PS-b-PMMA film. It has been previously shown that matching a calculated SSEF within the polymer film to the experimental Raman amplitude versus incident angle data can be used to measure the polymer film's thickness when its refractive index is known. ${ }^{24,25,37}$ For the PS-b-PMMA and PS-bPMMA:PMMA films the refractive index is not known at the relevant excitation wavelength for these studies. More generally, there are many mixed polymer films where the index of refraction is not known. However, since the Raman amplitude ratio encodes the fractional composition for the mixed polymer film, it can be used to calculate a refractive index for the polymer film. The Raman amplitude ratio is calculated using equation 2 for each incident angle of light, and then an average over all incident angles is calculated. Finally, the average Raman amplitude ratio is used 
in equation 4 to determine the polymer film's refractive index $\left(\mathrm{RI}_{\text {film }}\right)$, where $R I_{P S}$ and $R I_{P M M A}$ are the indices of refraction for the homopolymers at the excitation wavelength.

$$
R I_{\text {film }}=\left(r_{P S} \times R I_{P S}\right)+\left(\left(1-r_{P S}\right) \times R I_{P M M A}\right)
$$

Table 1: The Raman amplitude ratios and indices of refraction for the prepared films determined by SA Raman as well as a thickness comparison between SA Raman and profilometry measurements.

\begin{tabular}{|c|c|c|c|c|c|}
\hline Sample & $\begin{array}{c}\text { Raman } \\
\text { Amplitude } \\
\text { Ratio }\left(\mathbf{r}_{\mathrm{PS}}\right)^{1}\end{array}$ & $\begin{array}{l}\text { Refractive } \\
\text { Index (RI) }\end{array}$ & $\begin{array}{c}\text { SA Raman } \\
\text { Thickness } \\
(\mathbf{n m})\end{array}$ & $\begin{array}{c}\text { Profilometry } \\
\text { Thickness (nm) }\end{array}$ & $\begin{array}{c}\text { Percent } \\
\text { Difference } \\
(\%)\end{array}$ \\
\hline PS-b-PMMA & $0.78 \pm 0.01$ & 1.558 & 550 & $495.4 \pm 0.8$ & 10 \\
\hline $\begin{array}{c}\text { 1:2 PS-b- } \\
\text { PMMA:PMMA }\end{array}$ & $0.32 \pm 0.01$ & 1.515 & 780 & $870 \pm 10$ & 10 \\
\hline $\begin{array}{c}\text { 1:2.4 PS-b- } \\
\text { PMMA:PMMA }\end{array}$ & $0.28 \pm 0.02$ & 1.511 & 790 & $850 \pm 70$ & 8 \\
\hline $\begin{array}{c}\text { 1:2.6 PS-b- } \\
\text { PMMA:PMMA }\end{array}$ & $0.27 \pm 0.01$ & 1.510 & 830 & $824 \pm 3$ & 0.8 \\
\hline $\begin{array}{c}\text { 1:3 PS-b- } \\
\text { PMMA:PMMA }\end{array}$ & $0.25 \pm 0.04$ & 1.509 & 920 & $936 \pm 5$ & 2 \\
\hline $\begin{array}{c}\text { 1:4 PS-b- } \\
\text { PMMA:PMMA }\end{array}$ & $0.236 \pm 0.006$ & 1.507 & 900 & $950 \pm 30$ & 6 \\
\hline $\begin{array}{c}97.0 \mathrm{mg} / \mathrm{mL} \\
\text { P2VN-b-PMMA }\end{array}$ & $0.29 \pm 0.01$ & 1.525 & 815 & $795 \pm 6$ & 2 \\
\hline $\begin{array}{c}133 \mathrm{mg} / \mathrm{mL} \\
\text { P2VN-b-PMMA }\end{array}$ & $0.29 \pm 0.01$ & 1.525 & 965 & $971 \pm 8$ & 0.6 \\
\hline
\end{tabular}

${ }^{1}$ This ratio is indicative of the fractional composition of the polymer film.

The refractive index determined from equation 4 is 1.558 for the PS-b-PMMA film. The SSEF calculated using the 1.558 refractive index and a thickness that minimized the residual between the fit and experimental data (black line in Figure 3B), shows a waveguide mode maximum angle at $56.04 \pm 0.08^{\circ}$ and a fwhm of $4.7 \pm 0.3^{\circ}$. The residual between the fit and 
experimental data is below $10 \%$. There is a $10 \%$ difference between the thickness determined by SA Raman spectroscopy and the value measured by profilometry (Table 1).

Figure 3C-L show the SA Raman data and $1001 \mathrm{~cm}^{-1}$ PS peak amplitude versus incident angle graphs for the 1:2, 1:2.4, 1:2.6, 1:3, and 1:4 PS-b-PMMA:PMMA films. Compared to the measurement on the PS-b-PMMA film, the PMMA Raman peak amplitude increases for the PSb-PMMA:PMMA films (Figure 3C, E, G, I. and K). This is consistent with the increasing fractional compositions of PMMA in the polymer films. There is a general decreasing trend in the magnitude of the Raman amplitude ratios $\left(\mathrm{r}_{\mathrm{PS}}\right)$ as more PMMA is added, although not all the values are statistically different (Table 1).

Table 2: Waveguide mode location and fwhm determined by fitting the $1001 \mathrm{~cm}^{-1}$ PS peak amplitude versus incident angle spectrum from SA Raman measurements.

\begin{tabular}{c|c|c} 
Sample & $\begin{array}{c}\text { Waveguide Mode } \\
\text { Location }\left({ }^{\circ}\right)\end{array}$ & $\begin{array}{c}\text { Waveguide Mode } \\
\text { fwhm }\left(^{\circ}\right)\end{array}$ \\
\hline PS-b-PMMA & $56.04 \pm 0.08$ & $4.7 \pm 0.3$ \\
\hline $\begin{array}{c}\text { 1:2 PS-b- } \\
\text { PMMA:PMMA }\end{array}$ & $56.06 \pm 0.03$ & $2.2 \pm 0.1$ \\
\hline $\begin{array}{c}\text { 1:2.4 PS-b- } \\
\text { PMMA:PMMA }\end{array}$ & $55.90 \pm 0.02$ & $2.06 \pm 0.06$ \\
\hline $\begin{array}{c}\text { 1:2.6 PS-b- } \\
\text { PMMA:PMMA }\end{array}$ & $56.12 \pm 0.02$ & $1.94 \pm 0.06$ \\
\hline $\begin{array}{c}\text { 1:3 PS-b- } \\
\text { PMMA:PMMA }\end{array}$ & $56.48 \pm 0.06$ & $1.7 \pm 0.2$ \\
\hline $\begin{array}{c}\text { 1:4 PS-b- } \\
\text { PMMA:PMMA }\end{array}$ & $56.46 \pm 0.02$ & $1.59 \pm 0.05$ \\
\hline 97.0 mg/mL P2VN-b- & & $2.10 \pm 0.03$ \\
PMMA & $56.85 \pm 0.01$ & $1.51 \pm 0.03$
\end{tabular}


The addition of PMMA homopolymer causes the waveguide mode's fwhm to narrow compared to the PS-b-PMMA film. Table 2 summarizes the waveguide mode properties for the prepared polymer films. These observed changes in the waveguide mode fwhm are generally indicative of an increase in the film thickness. However, varying the polymer fractional composition also causes a change in the refractive index compared to the PS-b-PMMA film, and this will also affect the waveguide properties. This highlights the need to simultaneously determine both the index of refraction and thickness for each polymer film.

The refractive index calculated by equation 4 using the experimental Raman amplitude ratios $\left(\mathrm{r}_{\mathrm{PS}}\right)$ for each PS-b-PMMA:PMMA film is shown in Table 1, and are used in the subsequent SSEF calculations to fit the experimental data. As with the PS-b-PMMA film, the best fit to the experimental data is determined by minimizing the residual between the fit and experimental data while changing the polymer film thickness. There is an average $5 \%$ difference between the thicknesses measured by SA Raman spectroscopy and profilometry for the PS-bPMMA film and five PS-b-PMMA:PMMA films (Table 1). The agreement between the calculated SSEF and the experimental data demonstrate that the film thickness can be accurately determined (i.e., provides a result consistent with the profilometry measurements) when the Raman amplitude ratio is used to determine the polymer film's refractive index.

In order to demonstrate the applicability of the method with other mixed polymer compositions that have distinct Raman peaks for each component, two P2VN-b-PMMA films are measured. The relative Raman cross section between P2VN and PMMA is experimentally determined and calculated using equation 1 . The relative Raman cross section is then used in determining the Raman amplitude ratio ( $\left.\mathrm{r}_{\mathrm{P} 2 \mathrm{VN}}\right)$ for the P2VN-b-PMMA films $(0.29 \pm 0.01)$, 
which is similar to the manufacturer's reported 0.27 monomer fraction. The refractive index for the P2VN-b-PMMA films is determined using equation 5, and is shown in table 1.

$$
R I_{\text {film }}=\left(r_{P 2 V N} \times R I_{P 2 V N}\right)+\left(\left(1-r_{P 2 V N}\right) \times R I_{P M M A}\right)(5)
$$
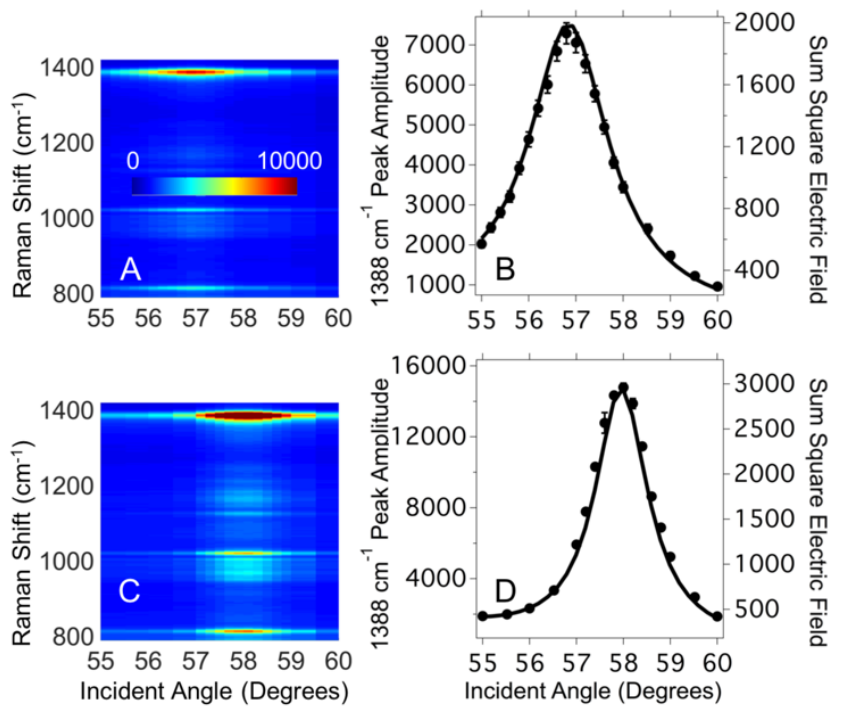

Figure 4: (A and C) SA Raman spectra of P2VN-b-PMMA films plotted on the same color amplitude scale showing 1388 and $812 \mathrm{~cm}^{-1}$ peaks of P2VN and PMMA, respectively. (B and D) $1388 \mathrm{~cm}^{-1}$ peak amplitude versus incident angle graph of the P2VN-b-PMMA films where the solid black line represents the SSEF fit to the experimental data (circles). (A and B) show the film formed by spin coating the $97.0 \mathrm{mg} / \mathrm{mL}$ solution and (C and D) show the film formed by spin coating the $133 \mathrm{mg} / \mathrm{mL}$ solution (as described in the Materials and Methods section).

Figure 4A and C shows the SA Raman spectra collected at each incident angle from $55^{\circ}$ to $60^{\circ}$. The $1388 \mathrm{~cm}^{-1} \mathrm{P} 2 \mathrm{VN}$ peak corresponds to a $\mathrm{CH}$ stretch mode and $812 \mathrm{~cm}^{-1}$ peak corresponds to PMMA's carbonyl stretching vibration mode. The P2VN peak is more intense 
compared to PMMA peak due to the $\mathrm{CH}$ stretching mode having a larger Raman cross section. Figure 4B and D show the $1388 \mathrm{~cm}^{-1}$ peak amplitude versus incident angle graph extracted from the data in Figure 4A and C. There is a shift in the waveguide mode location and fwhm when comparing the $\mathrm{P} 2 \mathrm{VN}$ peak from the two films prepared by spin coating different solution concentrations of the block copolymer. The $97.0 \mathrm{mg} / \mathrm{mL}$ solution forms a $795 \pm 6 \mathrm{~nm}$ thick film (Table 1) with a waveguide mode at $56.85^{\circ} \pm 0.01^{\circ}$ and a fwhm of $2.10^{\circ} \pm 0.03^{\circ}$ (Table 2). The waveguide mode location shifts to a higher angle $\left(57.95^{\circ} \pm 0.01^{\circ}\right)$ and with a smaller fwhm $\left(1.51^{\circ} \pm 0.03^{\circ}\right)$ for the film prepared from the high concentration solution due to the resulting thicker film (971 $\pm 8 \mathrm{~nm})$. The differences in the SA Raman data for the two P2VN-b-PMMA films is due only to the change in the thickness since both films have the same Raman amplitude ratio $\left(\mathrm{r}_{\mathrm{P} 2 \mathrm{VN}}\right)$ and refractive index. There is a $0.6 \%$ and $2 \%$ difference between the thicknesses determined by SA Raman and profilometry (Table 1) for the respective films.

\section{Conclusion}

For PS-b-PMMA, PS-b-PMMA:PMMA, and P2VN-b-PMMA films with unknown refractive index and thickness, both parameters can be extracted from the SA Raman data set. The refractive index is calculated from the fractional composition as measured by the SA Raman peak amplitude ratio and the known refractive index of the homopolymers. The thickness is extracted from fitting the $1001 \mathrm{~cm}^{-1} \mathrm{PS}$ or $1388 \mathrm{~cm}^{-1} \mathrm{P} 2 \mathrm{VN}$ peak amplitude versus incident angle using the determined refractive index and SSEF calculations. This measurement does not require an external probe (as required for the fluorescence analysis) and is not destructive (in contrast to the profilometry measurement). A "real" application of the SA Raman method will neither require the use of a fluorescent marker nor profilometry and is thus nondestructive and requires 
little sample preparation. This technique provides thicknesses for films with an average 5\% difference between profilometry, and on polymer films that are generally too thin for many traditional Raman spectroscopy, as well as other optical thickness measurements. PS-b-PMMA, PS-b-PMMA:PMMA, and P2VN-b-PMMA films were used to demonstrate the method, which will be fully compatible with a range of other mixed polymer systems. SA Raman spectroscopy will be useful whenever the components have distinguishable Raman peaks for measuring films used in energy capture and conversion devices, monitoring films prepared for microelectronics and optics, and biomedical applications. 


\section{Acknowledgement}

This research was supported by the U.S. Department of Energy (DOE), Office of Science, Basic Energy Sciences, Chemical Sciences, Geosciences, and Biosciences Division. The research was performed at the Ames Laboratory, which is operated for the U.S. DOE by Iowa State University under contract \# DE-AC02-07CH11358.

\section{References}

(1) Lin, Y.; Zhao, F.; He, Q.; Huo, L.; Wu, Y.; Parker, T. C.; Ma, W.; Sun, Y.; Wang, C.; Zhu, D.; Heeger, A. J.; Marder, S. R.; Zhan, X. JACS 2016, 138, 4955-4961.

(2) He, Z.; Xiao, B.; Liu, F.; Wu, H.; Yang, Y.; Xiao, S.; Wang, C.; Russell, T. P.; Cao, Y. Nat Photon 2015, 9, 174-179.

(3) Mori, D.; Benten, H.; Okada, I.; Ohkita, H.; Ito, S. Energy \& Environmental Science 2014, 7, 2939-2943.

(4) Paterson, A. F.; Treat, N. D.; Zhang, W.; Fei, Z.; Wyatt-Moon, G.; Faber, H.; Vourlias, G.; Patsalas, P. A.; Solomeshch, O.; Tessler, N.; Heeney, M.; Anthopoulos, T. D. Adv. Mater. 2016, 28, 7791-7798.

(5) Landi, G.; Altavilla, C.; Ciambelli, P.; Neitzert, H. C.; Iannace, S.; Sorrentino, A. AIP Conference Proceedings 2015, 1695, 020044.

(6) Wang, X.; Lee, W. H.; Zhang, G.; Wang, X.; Kang, B.; Lu, H.; Qiu, L.; Cho, K. Journal of Materials Chemistry C 2013, 1, 3989-3998.

(7) Rezem, M.; Kelb, C.; Günther, A.; Rahlves, M.; Reithmeier, E.; Roth, B., 2016, pp 975112975112-975116.

(8) Fujii, R.; Guo, J.; Klepp, J.; Pruner, C.; Fally, M.; Tomita, Y. Opt. Lett. 2014, 39, 3453-3456.

(9) Ladj, R.; Bitar, A.; Eissa, M. M.; Fessi, H.; Mugnier, Y.; Le Dantec, R.; Elaissari, A. Int. J.

Pharm. 2013, 458, 230-241.

(10) Guo, B.; Glavas, L.; Albertsson, A.-C. Prog. Polym. Sci. 2013, 38, 1263-1286.

(11) Ng, A.; Li, C. H.; Fung, M. K.; Djurišić, A. B.; Zapien, J. A.; Chan, W. K.; Cheung, K. Y.; Wong, W.-Y. The Journal of Physical Chemistry C 2010, 114, 15094-15101.

(12) Hilfiker, J. N.; Stadermann, M.; Sun, J.; Tiwald, T.; Hale, J. S.; Miller, P. E.; AracneRuddle, C. Appl. Surf. Sci.

(13) Bobbitt, J. M.; Weibel, S. C.; Elshobaki, M.; Chaudhary, S.; Smith, E. A. Anal. Chem. 2014, 86, 11957-11961.

(14) Zhong, Y.; Suzuki, K.; Inoue, D.; Hashizume, D.; Izawa, S.; Hashimoto, K.; Koganezawa, T.; Tajima, K. Journal of Materials Chemistry A 2016, 4, 3335-3341.

(15) Rohini, R.; Bose, S. ACS applied materials \& interfaces 2014, 6, 11302-11310.

(16) Cha, K. J.; Lih, E.; Choi, J.; Joung, Y. K.; Ahn, D. J.; Han, D. K. Macromolecular bioscience 2014, 14, 667-678.

(17) Lindqvist, C.; Sanz-Velasco, A.; Wang, E.; Bäcke, O.; Gustafsson, S.; Olsson, E.; Andersson, M. R.; Müller, C. Journal of Materials Chemistry A 2013, 1, 7174-7180. 
(18) Lee, J.; Kang, M. H.; Lim, W. C.; Shin, K.; Lee, Y. Surf. Interface Anal. 2013, 45, 498-502. (19) Lee, Y.; Lee, J.; Lim, W. C.; Shin, K.; Kim, K.-J. Surf. Interface Anal. 2011, 43, 277-280.

(20) Tran, W.; Tisinger, L. G.; Lavalle, L. E.; Sommer, A. J. Appl. Spectrosc. 2015, 69, 230-238.

(21) Nikonenko, N.; Tretinnikov, O. J. Appl. Spectrosc. 2008, 75, 878-882.

(22) Yang, P.; Meng, X.; Zhang, Z.; Jing, B.; Yuan, J.; Yang, W. Anal. Chem. 2005, 77, 10681074.

(23) Chan, K. L. A.; Kazarian, S. G. Appl. Spectrosc. 2003, 57, 381-389.

(24) Matthew, W. M.; Vy, H. T. N.; Emily, A. S. Vib. Spectrosc 2013, 65.

(25) Damin, C. A.; Nguyen, V. H.; Niyibizi, A. S.; Smith, E. A. Analyst 2015, 140, 1955-1964.

(26) Meyer, M. W.; Larson, K. L.; Mahadevapuram, R. C.; Lesoine, M. D.; Carr, J. A.;

Chaudhary, S.; Smith, E. A. ACS applied materials \& interfaces 2013, 5, 8686-8693.

(27) Tsai, H.; Pitera, J. W.; Miyazoe, H.; Bangsaruntip, S.; Engelmann, S. U.; Liu, C.-C.; Cheng, J. Y.; Bucchignano, J. J.; Klaus, D. P.; Joseph, E. A.; Sanders, D. P.; Colburn, M. E.; Guillorn, M. A. ACS Nano 2014, 8, 5227-5232.

(28) Osinkina, L.; Lohmüller, T.; Jäckel, F.; Feldmann, J. The Journal of Physical Chemistry C 2013, 117, 22198-22202.

(29) Albrecht, T. R.; Bedau, D.; Dobisz, E.; He, G.; Grobis, M.; Hellwig, O.; Kercher, D.; Lille, J.; Marinero, E.; Patel, K.; Ruiz, R.; Schabes, M. E.; Lei, W.; Weller, D.; Tsai-Wei, W.

Magnetics, IEEE Transactions on 2013, 49, 773-778.

(30) Cho, W. J.; Kim, Y.; Kim, J. K. ACS Nano 2011, 6, 249-255.

(31) Jung, Y. S.; Lee, J. H.; Lee, J. Y.; Ross, C. A. Nano Lett. 2010, 10, 3722-3726.

(32) Schneider, G. F.; Calado, V. E.; Zandbergen, H.; Vandersypen, L. M. K.; Dekker, C. Nano

Lett. 2010, 10, 1912-1916.

(33) McKee, K.; Smith, E. The Review of scientific instruments 2010, 81, 43106.

(34) Kasarova, S. N.; Sultanova, N. G.; Ivanov, C. D.; Nikolov, I. D. Opt. Mater. 2007, 29, 1481-1490.

(35) Bass, M.; DeCusatis, C.; Enoch, J.; Lakshminarayanan, V.; Li, G.; MacDonald, C.;

Mahajan, V.; Van Stryland, E. Handbook of Optics, Third Edition Volume IV: Optical Properties of Materials, Nonlinear Optics, Quantum Optics (set); Mcgraw-hill, 2009.

(36) Sultanova, N.; Kasarova, S.; Nikolov, I. Acta Physica Polonica-Series A General Physics 2009, 116, 585.

(37) Lesoine, M. D.; Bobbitt, J. M.; Zhu, S.; Fang, N.; Smith, E. A. Anal. Chim. Acta 2014, 848, 61-66. 


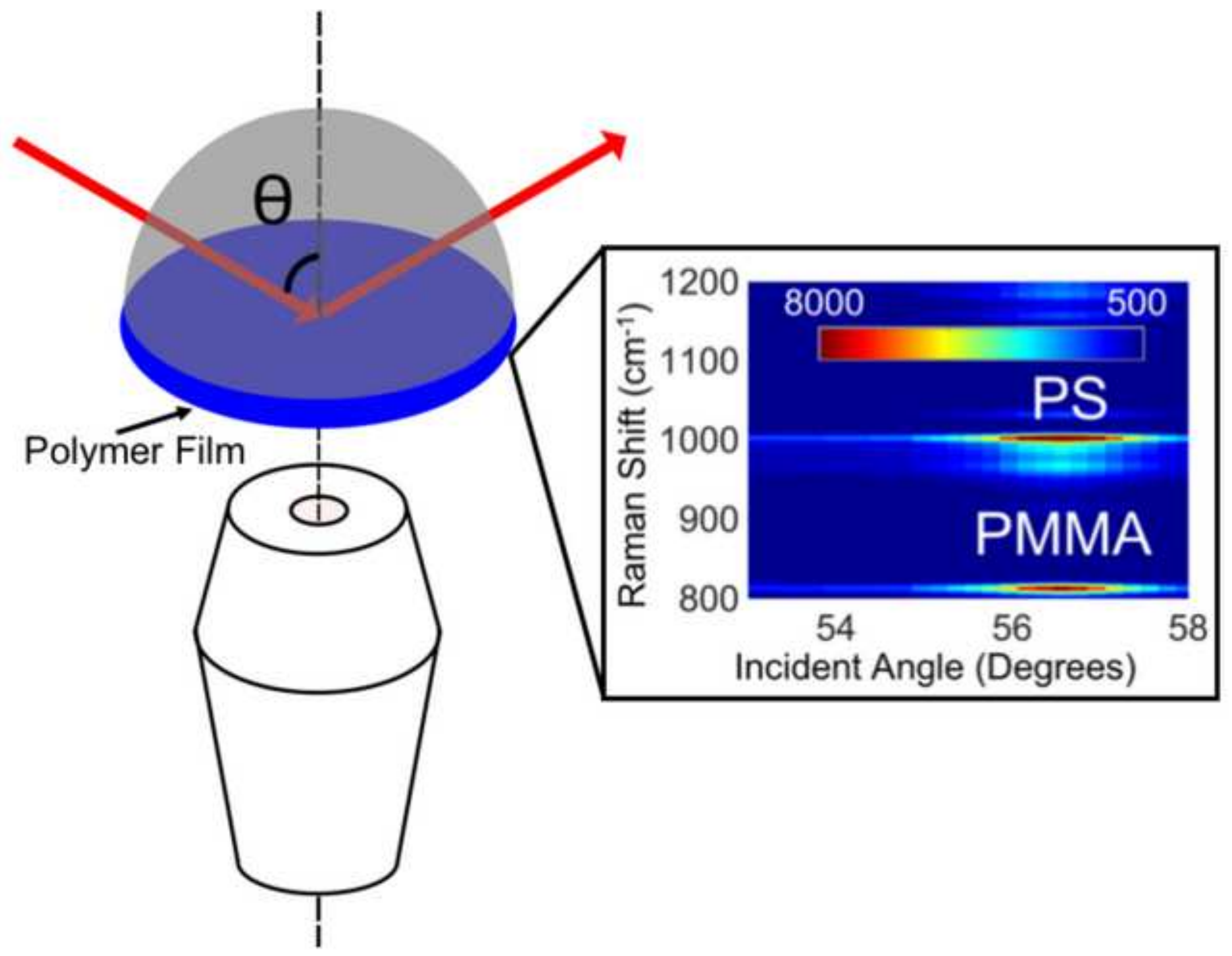

